Esta revista forma parte del acervo de la Biblioteca Jurídica Virtual del Instituto de Investigaciones Jurídicas de la UNAM http://www.juridicas.unam.mx 


\title{
Hacia una coordinación efectiva entre regímenes especializados para garantizar una interpretación armoniosa en el derecho internacional público moderno
}

\section{Towards Effective Coordination between Specialized Regimes to Guarantee a Harmonious Interpretation in Modern Public International Law}

Vers une coordination efficace entre les régimes spécialisés pour garantir une interprétation harmonieuse du droit international public moderne

\author{
Gerhard Niedrist* \\ Aida Figueroa Bello**
}

SUMARIO: I. Introducción. II. Características de solución de controversias en regímenes especializados. III. Conflictos entre regímenes en el derecho internacional moderno. IV. Coordinación entre regímenes especializados en derecho internacional público. V. A manera de conclusión. VI. Bibliografía.

* Instituto Tecnológico y de Estudios Superiores de Monterrey, México; ORCID ID: https: / /orcid.org/0000-0002-4245-0881,gerhard.niedrist@tec.mx.

** Universidad Autónoma de Nuevo León, México; ORCID ID: https: / /orcid.org/0000-000 2-2699-642X.

Artículo recibido el 27 de mayo de 2020 Aprobado para publicación el 9 de octubre de 2020 
RESUMEN: El derecho internacional moderno se caracteriza, en gran parte, por sus regímenes especializados. El derecho comercial internacional, la protección internacional de derechos humanos y el derecho ambiental internacional son sólo algunos de los ejemplos más importantes de áreas aisladas en el derecho internacional, mismas que se caracterizan por su reclamo de hegemonía. El presente artículo analiza la interacción entre los diferentes regímenes, concluyendo así que el derecho internacional actual carece de las capacidades para coordinar de manera efectiva tales regímenes. En este mismo sentido, argumentaremos que resulta imprescindible una necesaria e imperiosa coordinación entre los diferentes mecanismos de solución de controversias. Una coordinación efectiva entre regímenes especializados se podrá llevar a cabo mediante reglas como el forum non conveniens del common law, y no a través de criterios de secuencia temporal, como lo establece la Convención de Viena sobre el Derecho de los Tratados.

Palabras clave: régimen, solución de controversias, fragmentación, common law.

ABSTRACT: Modern international law is largely characterized by its specialized regimes. International commercial law, international protection of human rights and international environmental law are only the most important examples of isolated areas in international law and which are characterized by the establishment of a certain hegemony of their rules. This article analyzes the interaction between different regimes and will conclude that current international law lacks the capacities to effectively coordinate these regimes. We will argue that coordination is required between the different dispute settlement mechanisms. An effective coordination between specialized regimes can be carried out through rules such as the forum non conveniens of common law and not, as established by the Vienna Convention on the Law of Treaties, through criteria of temporal sequence.

Key words: regime, dispute resolution, fragmentation, common law.

RÉSUMÉ: Le droit international moderne se caractérise, dans une large mesure, par ses régimes spécialisés. Le droit commercial international, la protection internationale des droits de l'homme et le droit international de l'environnement ne sont que quelques-uns des exemples les plus importants de domaines isolés du droit international, qui se caractérisent par leur prétention à l'hégémonie. Cet article analyse l'interaction entre les différents régimes, concluant ainsi que le droit international actuel n'a pas les capacités de coordonner efficacement ces régimes. Dans ce même sens, nous soutiendrons qu'une coordination nécessaire et impérative entre les différents mécanismes de règlement des différends est essentielle. Une coordination efficace entre les régimes spécialisés peut être assurée par des règles telles que le forum non conveniens de common law, et non plus par des critères de chronologie, comme établi par la Convention de Vienne sur le Droit des Traités.

Mots-clés: régime, règlement des différends, fragmentation, common law. 


\section{INTRODUCCIÓN}

El derecho internacional público ha sido durante mucho tiempo un ámbito jurídico materialmente limitado, enfocándose de manera preponderante a cuestiones de derecho del mar, acuerdos comerciales, relaciones consulares y diplomáticas, regulación de fronteras, así como a acuerdos de paz y reparaciones. Los únicos sujetos eran los Estados y sus métodos de solución de controversias se limitaban a métodos diplomáticos y políticos. ${ }^{1}$

Como es bien sabido, la transformación hacia el derecho internacional moderno comenzó después de la Primera Guerra Mundial, cuando los Estados dieron origen a las organizaciones internacionales con personalidad propia. La Sociedad de las Naciones - como antecesora de las Naciones Unidas - hizo los primeros intentos en limitar el uso de la fuerza y estableció con la Corte Permanente de Justicia Internacional - el primer tribunal permanente para la resolución de conflictos entre Estados. Organizaciones como la Organización Internacional del Trabajo, la Unión Postal Universal y la Unión Internacional de Telecomunicaciones son los primeros ejemplos de especialización en derecho internacional.

Dicha transformación se intensificó y, al menos en parte, concluyó después de la Segunda Guerra Mundial, cuando - en conjunto y en el entorno de la fundación de las Naciones Unidas - surgieron diversas agencias especializadas y otras numerosas organizaciones internacionales. Hoy en día, las organizaciones internacionales se ocupan de un sinfín de temas, como agricultura, cuestiones humanitarias, medio ambiente, pesca, cuestiones marítimas, control de armas, energía, finanzas, comercio y aduanas, cooperación policial, derechos humanos, transporte, integración regional, educación y cultura, entre otros. Y ha sido así que de esta manera se han desarrollado diferentes regímenes de derecho internacional, los cuales regulan y abarcan ciertas áreas temáticas especializadas mediante un conjunto de principios, normas, reglas y procesos de toma de decisiones. ${ }^{2}$

1 La excepción sería el arbitraje, el cual surgió a finales del siglo XIX.

2 Bradford, Anu, "Regime Theory", en Wolfrum, Rüdiger (comp.), The Max Planck Encyclopedia of Public International Law, Oxford, Oxford University Press, 2012, vol. 8, pp. 737-743, en particular p. 737. 
No obstante lo anterior, la transformación y los cambios en el derecho internacional público no sólo se han basado en la ampliación y especialización del derecho sustantivo, sino también en cambios trascendentales y fundamentales en el derecho procesal internacional. Ello ha sido así que algunas de las nuevas organizaciones internacionales han creado sus propios y especializados organismos de solución de controversias. De los ejemplos más importantes, podemos citar al órgano de solución de controversias de la Organización Mundial del Comercio (OMC), diversos tribunales y órganos cuasijudiciales de la protección internacional de derechos humanos, así como otros tribunales y órganos de solución de controversias internacionales y regionales. ${ }^{3}$

Resulta por demás interesante señalar que una de las consecuencias de la especialización del derecho internacional público es precisamente su fragmentación. Ello implica que en el seno de un régimen especializado se encuentran tanto derecho sustantivo como reglas procesales de solución de controversias. Tales regímenes tienen mínimos puntos de enlace entre sí, y aún menos hacia el derecho internacional general.

El presente artículo examina la coordinación entre regímenes especializados en el derecho internacional, cuyo análisis se centra en el derecho comercial internacional de la OMC y la protección internacional de los derechos humanos que, además de contar con una cobertura casi global, también han establecido sistemas de solución de controversias de gran alcance y enorme trascendencia. Por su parte, el régimen de protección internacional del medio ambiente no cuenta con un sistema homogéneo de solución de controversias, pero sí con importantes puntos de conflicto con el sistema de comercio internacional.

En este sentido, argumentaremos que organismos integradores del derecho internacional público, tales como las Naciones Unidas, o bien su Corte Internacional de Justicia (CIJ), están perdiendo influencia y capacidad de coordinación entre diferentes áreas del derecho internacional ante la creciente especialización y fragmentación de dicho orden jurídico internacio-

3 Entre muchos otros, se encuentran el Tribunal Europeo de Derechos Humanos, la Corte Interamericana de Derechos Humanos, la Corte Africana de Derechos Humanos y de los Pueblos, los comités de los tratados universales de derechos humanos, la Corte Penal Internacional y tribunales penales para ex-Yugoslavia y Ruanda, el Tribunal de Justicia de la Unión Europea, el Tribunal de Justicia Andino o el Tribunal Internacional del Derecho del Mar. 
nal. ${ }^{4}$ De igual modo, tampoco la Convención de Viena sobre el Derecho de los Tratados (CVDT o Convención de Viena) puede ejercer tales facultades, dado que ésta tiene una visión homogénea del derecho internacional, cuyos criterios de coordinación se basan en lineamientos temporales, y no atiende a la lógica de los emergentes regímenes especializados.

No obstante, en la jurisprudencia de los órganos de solución de controversias de los regímenes especializados podemos observar una creciente práctica hacia una interpretación armoniosa en derecho internacional. Asimismo, y dada la debilidad de la CVDT, sólo los elementos de derecho procesal nacional — basado en derecho consuetudinario y principios generales de derecho - pueden brindar dicha coordinación. El common law - en claro contraste a los sistemas de derecho civil — se enfoca a criterios de especialidad, permitiendo así litigios paralelos, y, por tanto, pareciera tener elementos más apropiados para proporcionar dicha interpretación armónica en el derecho internacional público.

\section{CARACTERÍSTICAS DE SOLUCIÓN DE CONTROVERSIAS EN REGÍMENES ESPECIALIZADOS}

Resulta interesante mencionar que a la OMC, a pesar de un evidente estancamiento en su más reciente desarrollo, se le considera un caso exitoso en el derecho internacional. Con sus 164 miembros tiene una cobertura casi global, con estructuras flexibles y efectivas, y con un sistema de solución de controversias rápido y eficaz; como dato, hasta la fecha, ha resuelto más de 500 casos, muchos más que en cualquier otro sistema global e internacional de solución de controversias. ${ }^{5}$

Por otro lado, la OMC recibe también constantes críticas debido a su relación indiferenciada — y, en parte, indefinida — hacia otras áreas del derecho internacional. En general, resulta indiscutible que la OMC constituye un régimen especializado en el derecho internacional. Sin embargo, tal pro-

4 Posner, Eric A., "The Decline of the International Court of Justice”, Olin Program in Law and Economics Working Paper No. 233, 2004.

5 Comparativamente, la CIJ ha decidido, desde 1945, 162 casos, mientras que el Tribunal Internacional del Derecho de Mar sólo 23 casos, desde 1996. 
nunciamiento no implica conocer en qué medida el derecho internacional general y el derecho de otros regímenes pueden ser aplicados dentro del sistema de la OMC. En particular, la relación del derecho de la OMC tanto con la protección internacional del medio ambiente como con el sistema internacional de los derechos humanos se discute ampliamente en la literatura académica. $^{6}$

Como es bien sabido, la solución de controversias dentro de regímenes especializados se caracteriza por su pretensión hegemónica. La OMC establece una hegemonía de carácter formal, la cual se basa, por un lado, en las disposiciones de su consideración de solución de controversias y, por el otro, en las características de los miembros de sus paneles y del Órgano de Apelaciones. Los miembros de los paneles no requieren ninguna competencia comprobada en ciencias jurídicas y, de igual modo, para los miembros del Órgano de Apelaciones, basta con tener una competencia en derecho comercial internacional, en particular sobre los acuerdos de la OMC.

Por ende, son expertos en comercio internacional, los cuales deciden cuestiones con efectos transversales hacia el derecho ambiental internacional o la protección internacional de los derechos humanos. Algunos ejemplos son las decisiones de la OMC sobre la explotación de tierras raras, ${ }^{7}$ sobre los métodos de pesca de atún en México, ${ }^{8}$ o bien el caso sobre la licenciación forzada de patentes contra VIH/SIDA (virus de inmunodeficiencia humana y síndrome de inmunodeficiencia adquirida, respectivamente). ${ }^{9}$ En conjunto, y con la gran aceptación del sistema de solución de contro-

6 Para un análisis detallado del derecho de la OMC, véanse Comisión de Derecho Internacional, "Fragmentation of International Law: Difficulties Arising from the Diversification and Expansion of International Law", Report of the Study Group of the International Law Commission, A/CN.4/L.682, 13 de abril de 2006, parás. 165-171; Böckenförde, Markus, "Zwischen Sein und Wollen - Über den Einfluss umweltvölkerrechtlicher Verträge im Rahmen eines WTOStreitbeilegungsverfahrens", Zeitschrift für allgemeines öffentliches Recht und Völkerrecht, vol. 63, 2003, pp. 971-1005.

7 World Trade Organization, China - Measures Related to the Exportation of Rare Earths, Tungsten, and Molybdenum, WT/DS431/R and Add.1 / WT/DS432/R and Add.1 / WT/DS433/R and Add.1, adopted 29.8.2014, upheld by Appellate Body Reports WT/DS431 / AB/R / WT / DS432/AB/R / WT/DS433/AB/R, pará. 7.149.

8 World Trade Organization, United States - Measures Concerning the Importation, Marketing and Sale of Tuna and Tuna Products, Panel Report, adopted 13.6.2012, WT/DS381/R, as modified by Appellate Body Report WT/DS381/AB/R, DSR 2012: IV, 2013.

9 Este caso finalmente no fue decidido de manera legal, sino a nivel político. 
Esta revista forma parte del acervo de la Biblioteca Jurídica Virtual del Instituto de Investigaciones Jurídicas de la UNAM

versias, el resultado es lo que Hestermeyer ha denominado "una jerarquía de facto" de la OMC hacia otros regímenes especializados de derecho internacional. ${ }^{10}$

Ahora bien, y hasta la fecha, ni los tribunales ni los órganos de protección internacional de derechos humanos han hecho referencia a los regímenes externos, y tampoco se ha desarrollado una discusión teórica respecto a dilucidar hasta qué grado los regímenes externos deben ser aplicables en el sistema de derechos humanos. En cambio, varios tribunales internacionales - en particular los europeos - han desarrollado una tendencia para verificar actos de organizaciones internacionales que resulten compatibles o relativos con derechos humanos internacionales. Tal es el caso de las sentencias Kadi, en las que el Tribunal de Justicia de la Unión Europea se reservó el derecho de revisar resoluciones del Consejo de Seguridad, ${ }^{11}$ y en las que el Comité de Derechos Humanos de las Naciones Unidas tomó una decisión claramente similar. ${ }^{12}$ En varias sentencias, tanto el Tribunal Europeo de Derechos Humanos como el Tribunal Constitucional alemán han exigido criterios y normas de derechos humanos dentro de organizaciones internacionales. ${ }^{13}$ En un caso sudafricano, compañías farmacéuticas tenían que retirar demandas ${ }^{14}$ contra licencias compulsivas de medicinas VIH/SIDA por la gran presión pública, dada la fuerte posición que particularmente tienen la salud pública y los derechos humanos en la Constitución. ${ }^{15}$ Con base en estos

10 Hestermeyer, Holger, Human Rights and the WTO: The Case of Patents and Access to Medicines, Oxford, Oxford University Press, 2007, pp. 193-197.

11 Tribunal de Justicia de la Unión Europea, Yassin Abdullah Kadi y Al Barakaat International Foundation vs. Council and Commission, (Kadi I), C-402/05P, Sentencia del 3 de septiembre de 2008, parás. 314 y 326; Tribunal de Justicia de la Unión Europea, Yassin Abdullah Kadi y Al Barakaat International Foundation vs. Council and Commission, (Kadi II), C-584/10P, Sentencia del 18 de julio de 2013, pará. 133.

12 Comité de Derechos Humanos, Nabil Sayadi y Patricia Vinck vs. Belgium, Communication No. 1472/2006, U.N. Doc. CCPR/C/94/D/1472/2006 (2008).

13 Bundesverfassungsgericht (Tribunal Constitucional Federal de Alemania), Eurocontrol, BVerfG, 23.06.1981 - 2 BvR 1107/77, 1124/77, 195/79; Tribunal Europeo de Derechos Humanos, Beer and Regan vs. Germany (Application no. 28934/95), 1999; Tribunal Europeo de Derechos Humanos, Waite and Kennedy vs. Germany (Application no. 26083/94), 1999.

14 High Court of South Africa, "Pharmaceutical Company Lawsuit (Forty-Two Applicants) against the Government of South Africa (Ten Respondents)", Notice of Motion in the High Court of South Africa (Transvaal Provincial Division), Case number: 4183/98.

15 Harrison, James, The Human Rights Impact of the World Trade Organisation, Portland, 2007, p. 158. 
ejemplos, podemos suponer que, en un caso ante un órgano de derechos humanos, las disposiciones del sistema internacional de derechos humanos prevalecerán sobre obligaciones de tipo comercial. Es importante mencionar que no se trata de una jerarquía de normas, sino "sólo" de una jerarquía en la aplicación entre diferentes regímenes. ${ }^{16}$

En otras palabras, al igual que la OMC, los derechos humanos y los demás regímenes especializados establecen una hegemonía de su propio sistema, esto es, prefieren aplicar su propio sistema sobre otros. En última instancia, son estas características inherentes a todos los regímenes especializados. El resultado es la fragmentación del derecho internacional, la cual, según varios presidentes de la CIJ, así como para algunos autores, pone en peligro la unidad del derecho internacional. Otras opiniones consideran a la fragmentación como un problema técnico, el cual surgió naturalmente de una creciente actividad y especialización del derecho internacional. ${ }^{17}$ En cualquier caso, la especialización y fragmentación del derecho internacional es un hecho, el cual resulta innegable, y una coordinación entre diferentes regímenes sólo podrá proveer el derecho internacional público general.

\section{CONFLiCTOS ENTRE REGÍMENES}

EN EL DERECHO INTERNACIONAL MODERNO

\section{Conflictos técnicos dentro de un régimen}

Es importante señalar que ningún sistema de solución de controversias se está desarrollando en completo aislamiento de otras áreas del derecho internacional público. Hasta la fecha, y de acuerdo con un estudio de Marceau, otros organismos internacionales de resolución de controversias se basaron en derechos de la OMC en más de 150 casos. ${ }^{18}$ Ello es una muestra de que la $\mathrm{OMC}$, a parte de su rapidez y flexibilidad interna, también ejerce una

16 Joseph, Sarah, Blame it on theWTO?: A Human Rights Critique, Oxford, Oxford University Press, 2011, p. 54.

17 Comisión de Derecho Internacional, op. cit., pará. 9o.

18 Marceau, Gabrielle et al., "The WTO's Influence on Other Dispute Settlement Mechanisms: A Lighthouse in the Storm of Fragmentation”, Journal of World Trade, vol. 47, núm. 3, 2013, pp. 481-574, en especial p. 481. 
considerable influencia en otros órganos de derecho internacional. Sin embargo, en un análisis detallado de tales casos se puede observar que las referencias hacia el sistema legislativo de la OMC hechas por órganos externos se limitan casi en su totalidad a casos de protección de inversiones, acuerdos comerciales regionales y protección de propiedad intelectual. Consecuentemente, de las 150 referencias, 42 se refieren a la Comunidad Andina, otras 31 al capítulo 19 del TLCAN (Tratado de Libre Comercio de América del Norte), 20 al CIADI (Centro Internacional de Arreglo de Diferencias relativas a Inversiones), 12 casos relativos al capítulo 11 del TLCAN, 9 al Mercosur y otros 8 al Centro de Arbitraje de la OMPI (Organización Mundial de la Protección Intelectual). ${ }^{19}$ Lo anterior supone que la influencia y el liderazgo de la OMC se limitan a su propio régimen de derecho comercial internacional, esto es, a aquellos organismos que ejercen iguales o similares facultades que la propia $\mathrm{OMC}$.

Así pues, conviene señalar que las razones por las cuales los órganos de solución de controversias recurren a la jurisprudencia de la OMC corresponden a, por un lado, que de esta manera pueden aclarar sus propios términos jurídicos, los cuales son iguales o similares a los de la OMC, y también, por otro lado, garantizar una interpretación armónica con el sistema legal de la OMC. Sin embargo, ninguno de estos sistemas, y tampoco la CVDT, exigen explícitamente el uso de derecho de la OMC. ${ }^{20}$

La protección internacional de los derechos humanos, que consiste en el sistema global y universal de derechos humanos en el entorno de las Naciones Unidas y en los tres sistemas regionales de los derechos humanos, funciona de manera similar. Frecuentemente, sus tribunales y órganos no sólo se basan en su propia jurisprudencia, sino que también tienen en cuenta a la de los demás sistemas. Al igual que con la OMC, el objetivo es nuevamente una interpretación uniforme del régimen global de derechos humanos sin ninguna obligación legal específica. ${ }^{21}$

19 Ibidem, pp. 484 y 531.

20 Ibidem, pp. 489 y 532; Petersmann, Ernst-Ulrich, The GATT/WTO Dispute Settlement System: International Law, International Organizations and Dispute Settlement, Leiden, Martinus Nijhoff, 1997, p. 48.

21 Lixinski, Lucas, "Treaty Interpretation by the Inter-American Court of Human Rights: Expansionism at the Service of the Unity of International Law", European Journal of International Law, vol. 21, núm. 3, 2010, pp. 585-604. 


\section{Característica de rupturas entre regímenes especializados}

Con respecto a conflictos entre regímenes de diferente enfoque y alcance, tal como, por ejemplo, entre la OMC, los derechos humanos y la protección internacional del medio ambiente, hemos de señalar que hasta la fecha han sido muy escasos.

Por lo que se refiere a casos relativos a la OMC con la protección internacional del medio ambiente, se han resuelto dentro de su propio sistema de solución de controversias de aquélla, mientras que conflictos relativos a derechos humanos han sido resueltos a un nivel político. Por otro lado, la OMC no gozaba de casi ninguna importancia en la resolución de conflictos de casos sobre medio ambiente o derechos humanos, lo cual al final implica un paralelismo de los diferentes regímenes especializados. No obstante, y en vista de la creciente fragmentación del derecho internacional, podemos suponer que en el futuro surgirá un mayor número de conflictos, quizá no de manera masiva en número, pero sí conflictos entre diferentes regímenes de derecho internacional. Son precisamente este tipo de casos para los cuales el derecho internacional público debe proveer y garantizar una función de coordinación.

En este contexto, es importante tener en cuenta que las diferencias no corresponden a dificultades técnicas legales o de jurisprudencia, las cuales dificultan la coexistencia armoniosa entre diferentes regímenes dentro de un marco general de derecho internacional, sino más bien a rupturas sociales e incompatibilidades en sus finalidades, que se reflejan en los diferentes regímenes. Rupturas entre el derecho de la OMC, los derechos humanos y el medio ambiente son naturales e inherentes al sistema, ya que ante situaciones iguales o parecidas, con diferentes bases legales y objetivos, se llega a conclusiones diferentes.

Por otro lado, resultan inexistentes tales rupturas entre regímenes y sistemas con objetivos afines y similares; un ejemplo de ello: entre los diferentes sistemas de derechos humanos en su relación con derecho humanitario o la preservación de la paz y seguridad internacional. ${ }^{22}$ Lo mismo aplica a la relación de la OMC con la protección de inversiones internacionales o acuerdos

22 Fischer-Lescano, Andreas y Teubner, Gunther, "Regime-Collisions: The Vain Search for Legal Unity in the Fragmentation of Global Law", Michigan Journal of International Law, vol. 25, 2004, pp. 999-1049, en especial pp. 1013 y 1014. 
comerciales regionales, donde todos, si bien tienen diferentes contenidos y prioridades, se enfocan al mismo objetivo de la liberalización del comercio. Sus conflictos son, en consecuencia, de carácter técnico, ${ }^{23}$ pero no per se incompatible. Por ende, la correspondiente jurisprudencia se enfoca más bien a conceptos técnicos procesales.

Por su parte, Pauwelyn describe conflictos entre dos regímenes modernos, pero parcialmente incompatibles, como conflictos duros (hard cases). ${ }^{24}$ Entre el derecho de la OMC, los derechos humanos y la protección internacional del medio ambiente, existe un conflicto de diferentes racionalidades dentro de un mundo globalizado moderno, ${ }^{25}$ basado en una fragmentación social causada por los diferentes objetivos de estos regímenes. Así, la función más importante que debe hacer en este contexto el derecho internacional es proporcionar un efecto de mediación y de equilibrio entre estos subsistemas fragmentados. ${ }^{26}$

Sin embargo, los conceptos sobre las características de tal coordinación y mediación se deben desarrollar de la misma manera, tal y como también se ha desarrollado en las últimas décadas el derecho internacional de regímenes especializados. Durante siglos, el derecho internacional fue dominado casi exclusivamente entre Estados ${ }^{27}$ y ejerció la función de coordinación entre ellos. En evidente contraste, el derecho internacional moderno, con su multitud de organismos internacionales especializados y actores no estatales, tiene una influencia reguladora más directa y determina cada vez más acciones administrativas y derecho administrativo a nivel global. No obstante, tal influencia administrativa global requiere, igualmente, de una coordinación constitucional globalizada para garantizar la coexistencia pacífica entre las diferentes realidades especializadas. ${ }^{28}$

23 Gantz, David A., "Dispute Settlement under the NAFTA and the WTO: Choice of Forum Opportunities and Risks for the NAFTA Parties", American University International Law Review, vol. 14, 1998, p. 1025.

${ }^{24}$ Pauwelyn, Joost, "The Role of Public International Law in the WTO: How Far Can We Go?”, The American Journal of International Law, vol. 95, núm. 3, 2001, pp. 535-578, en especial p. 545 .

25 Fischer-Lescano, Andreas y Teubner, Gunther, op. cit., p. 1017.

26 Ibidem, p. 1045.

27 Ibidem, p. 1008; Kuo, Ming-Sung, "Between Fragmentation and Unity: The Uneasy Relationship between Global Administrative Law and Global Constitutionalism”, San Diego International Law Journal, vol. 10, 2008, pp. 439-468, en particular p. 447.

28 Ibidem, p. 440. 
De acuerdo con un estudio de la Comisión de Derecho Internacional sobre la fragmentación en derecho internacional, dicha coordinación debe cumplir con al menos dos tareas principales, que a continuación se mencionan. Primero, se deben resolver los conflictos que surjan cuando órganos de solución de controversias de diferentes regímenes se contradicen en un mismo caso. En última instancia, un equilibrio entre este tipo de conflictos solamente se puede lograr con base en el derecho internacional general, el cual debe garantizar una coexistencia pacífica entre diferentes regímenes especializados. Segundo, y desde la perspectiva del derecho material, estas reglas de coordinación requieren una aceptación en la medida en que puedan ejercer su papel coordinador entre regímenes especializados que permitan un desarrollo armónico y paralelo entre diferentes normas especializadas. ${ }^{29}$

\section{Elementos hacia una interpretación armónica en derecho internacional}

Ante ello, y a pesar de que hasta la fecha surgieron pocos casos de conflicto entre regímenes no afines, resulta evidente la necesidad de reglas claras y específicas de coordinación entre las diferentes áreas especializadas. Pero ¿qué elementos debe tener una coordinación efectiva entre diferentes regímenes del derecho internacional público? En el caso Indonesia - Certain Measures Affecting the Automobile Industry, un panel de la OMC expresó la suposición de que los Estados normalmente no quieren intervenir en obligaciones que están en conflicto. ${ }^{30}$ Es esa la razón por la que la OMC usa tratados externos como fuentes interpretativas dentro de su propio sistema especializado, aunque en la aplicación dan preferencia a su propio sistema jurídico. En el caso Gabč́ḱkovo-Nagymaros de la CIJ, el juez Weeramantry, con referencia hacia la OMC, hizo un llamamiento hacia una interpretación armónica con el derecho internacional del medio ambiente. ${ }^{31}$ Este uso de elementos externos por órganos de solución de controversias especializados puede explicarse

29 Comisión de Derecho Internacional, op. cit., parás. 56-122.

30 "[T]here is a presumption against conflicts in that parties do not normally intend to incur conflicting obligations". WTO, Indonesia - Certain Measures Affecting the Automobile Industry, Panel Report, adopted 23.7.1998, WT/DS54/R, WT/DS55/R, WT/DS59/R, WT/ DS64/R, Corr. 1 and Corr. 2, Corr. 3 and Corr. 4, DSR 1998:VI, 2201, pará. 5.349.

31 Corte Internacional de Justicia, "Case concerning the Gabčíkovo-Nagymaros Project (Hungary vs. Slovakia) Separate Opinion of Vice-President Weeramantry”, ICJ Reports, 1997, 7. 
por un cierto deseo de armonía y coherencia entre diferentes sistemas. ${ }^{32}$ Sin embargo, tal anhelo hacia una coherencia carece de un fundamento jurídico, aun cuando pareciere que los organismos tratan de evitar inconsistentes y contradictorias interpretaciones. ${ }^{33}$

Tal y como detallan Fischer-Lescano y Teubner, la autonomía de regímenes se incrementa frente a otras áreas de derecho internacional, en cuanto más confían en la fuerza de su propia estructura institucional. ${ }^{34}$ Esto ocurre debido a que los órganos de aquellos regímenes tienden a ver a su propio sistema como integral y completo, y sólo recurren hacia el derecho internacional en general en situaciones excepcionales. ${ }^{35}$ Con ello, se explica el entendimiento hegemónico de la OMC, el cual llega a la comprensión de ésta como un sistema cerrado.

Sin embargo, la OMC también ha reconocido el derecho a una interpretación pacífica y armónica; en efecto, en su caso Korea - Diary, el Órgano de Solución de Controversias expresó:

In light of the interpretive principle of effectiveness, it is the duty of any treaty interpreter to "read all applicable provisions of a treaty in a way that gives meaning to all of them, harmoniously". An important corollary of this principle is that a treaty should be interpreted as a whole, and, in particular, its sections and parts should be read as a whole. Agreement expressly manifests the intention of the Uruguay Round negotiators that the provisions of the WTO Agreement and the Multilateral Trade Agreements included in its Annexes 1, 2 and 3 must be read as a whole. ${ }^{36}$

Es importante señalar que el Órgano de Apelaciones en esta decisión se refirió a la interpretación armoniosa dentro de la propia OMC, que se basa en un acuerdo marco que, a su vez, combina y coordina varios acuerdos sus$\operatorname{tantivos}^{37}$ con elementos del derecho procesal. ${ }^{38}$

32 Marceau, Gabrielle et al., op. cit., p. 490.

33 Ibidem, pp. 492 y 493.

34 Fischer-Lescano, Andreas y Teubner, Gunther, op. cit., pp. 1015 y 1016.

35 Simma, Bruno y Pulkowski, Dirk, "Of Planets and the Universe: Self-Contained Regimes in International Law”, European Journal of International Law, vol. 17, núm. 3, 2006, pp. 483-529, en especial p. 505.

36 World Trade Organization, Korea - Definitive Safeguard Measure on Imports of Certain Dairy Products, Report of the Appellate Body, adopted 14.12.1999, WT/DS98/AB/R, pará. 81.

37 Por ejemplo, de liberalización de comercio en bienes (GATT) y servicios (GATS) o la protección de propiedad intelectual (TRIPS).

38 Las reglas de solución de controversias se encuentran en el DSB. 
Resulta incomprensible ¿por qué este principio sólo debería ser aplicado dentro de la OMC?, sobre todo tomando en cuenta que no constituye un sistema cerrado, en completo aislamiento del resto del derecho internacional. En tal sentido, no se pudiera leer ni interpretar, sin las intenciones de sus partes, fuera de la misma. De tal modo que no resultaría factible que dos o más Estados quieran cooperar y proteger, ya sea en materia de medio ambiente o de derechos humanos, mediante sus respectivos tratados y, a la vez, que en Ginebra ignoren estos compromisos en sus procedimientos de solución de controversias ante la OMC.

Cabe destacarse que a partir de la jurisprudencia del Órgano de Apelaciones, igualmente, se puede deducir que la función de lex specialis es, en última instancia, una técnica para un equilibrio entre diferentes obligaciones contractuales. Por ejemplo, en el caso US - Gasoline, el Órgano de Solución de Controversias expresó, asimismo, que las excepciones no deben usarse de manera que frustren por completo los derechos contemplados en el GATT. ${ }^{39}$ En este mismo sentido se expresó el Órgano de Apelaciones en el caso US - Shrimp: "balance must be made between the right... to invoke an exception and the duty... to respect the treaty rights of the other members". ${ }^{40}$

En este contexto, es importante recordar dos conceptos básicos: primero, si bien los Estados que participan en regímenes especializados, como la OMC, la protección del medio ambiente y los derechos humanos, no son $100 \%$ idénticos, a grandes rasgos sí son los mismos; ${ }^{41}$ segundo, las reglas de interpretación del derecho internacional convencional no son automatismos que sólo estén sujetos a una secuencia cronológica. Eso significa que tanto la adaptación como la modificación del derecho no se originan basadas en algunas disposiciones lógicas y abstractas, sino se trata más bien de una au-

39 World Trade Organization, United States - Standards for Reformulated and Conventional Gasoline, Report of the Appellate Body, adopted 20.5.1996, WT/DS2/AB/R, pará. 21; Van Damme, Isabelle, Treaty Interpretation by theWTO Appellate Body, Oxford-Nueva York, Oxford University Press, 2009, p. 286.

40 World Trade Organization, United States - Import Prohibition of Certain Shrimp and Shrimp Products, Panel Report, adopted 6.11.1998, WT/DS58/R and Corr. 1, as modified by Appellate Body Report WT/DS58/AB/R, DSR 1998:VII, pará. 156.

41 Pauwelyn, Joost, "Bridging Fragmentation and Unity: International Law as a Universe of Inter-Connected Islands”, Michigan Journal of International Law, vol. 25, 2003, pp. 903-916, en especial pp. 903 y 904. 
torización del legislador para adaptar el derecho a través de nuevas reglas interpretativas. ${ }^{42}$

En el mismo sentido, no resulta argumentable, salvo en casos excepcionales, que los Estados quieran excluirse intencionalmente de la participación de regímenes especializados. Como bien sabemos, el régimen de la OMC se basa en el GATT de 1947 como simple tratado internacional, pero cuyas reglas, incluyendo excepciones del medio ambiente, fueron incorporadas casi de manera idéntica a la OMC en su fundación en 1995. Con una estricta interpretación temporal, los compromisos del GATT de 1947 quedarían limitados por el derecho del medio ambiente, emergido en los setenta y ochenta, y este último, por la OMC de 1995.

Atendiendo a todo lo anterior, pareciera que el derecho internacional público está desarrollando una costumbre hacia un desarrollo armónico entre regímenes especializados. Si efectivamente eso sucede, ello dependerá si se sigue estableciendo la práctica estatal ya existente, al igual que si los Estados, así como los demás sujetos de derecho internacional público, desarrollan la correspondiente opinio iuris.

\section{COORDINACIÓN ENTRE REGÍMENES ESPECIALIZADOS}

EN DERECHO INTERNACIONAL PÚBLICO

\section{1. ¿Coordinación por el derecho internacional general} (ONU, CIJ, Convención de Viena)?

En varias ocasiones se ha atribuido a las Naciones Unidas una función coordinadora e integradora como "Constitución del derecho internacional". ${ }^{43} \mathrm{Tal}$ autoridad se basa, sobre todo, en su alcance universal con una membresía de casi todos los países en el mundo, así como de los efectos coercitivos que tienen las resoluciones por parte del Consejo de Seguridad como encargado primordial para mantener la paz y la seguridad internacionales. Sin embargo,

42 Vranes, Erich, “Lex superior, lex specialis, lex posterior - Zur Rechtsnatur der «Konfliktlösungsregeln»”, Zeitschrift für ausländisches öffentliches Recht undVölkerrecht, vol. 65, 2005, pp. 391-405, en especial p. 397.

43 Fassbender, Bardo, "The United Nations Charter as Constitution of the International Community”, Columbia Journal of Transnational Law, vol. 36, 1998, pp. 529-619. 
ello significa que las Naciones Unidas solamente ejercen su liderazgo dentro de su propio régimen respecto a la pacífica y armónica convivencia entre Estados, careciendo de las facultades para ejercer tales funciones entre regímenes especializados del derecho internacional moderno. Aunado a ello, las Naciones Unidas se basan — particularmente el Consejo de Seguridad - en decisiones de índole material y política, las cuales no son aptas para establecer una coordinación formal-procesal entre regímenes especializados. Estas decisiones sistémicamente están en contra de la necesidad de dicha coordinación judicial.

Entre tanto, y como órgano judicial de las Naciones Unidas, la CIJ podría desempeñar ese papel de coordinación; sin duda alguna, es uno de los órganos de solución de controversias más importantes del derecho internacional general, que goza de reconocimiento y aceptación legal a nivel mundial. Sin embargo, el alcance de su jurisdicción está limitado, dado que sólo 72 de 193 Estados la reconocen plenamente, mediante declaraciones unilaterales, y en sus setenta y cinco años de existencia, la CIJ ha decidido sólo unos 120 casos. Además, la CIJ carece de flexibilidad en sus procedimientos, característica que es inherente a los nuevos regímenes especializados. De acuerdo con el nuevo artículo 34 del Estatuto de la Corte Internacional de Justicia, los procedimientos ante la CIJ están abiertos sólo para Estados, mientras que los procedimientos ante regímenes especializados también incluyen zonas aduaneras (OMC), individuos (derechos humanos) y grupos de interés (derecho ambiental). Si bien es cierto que se propuso de lege ferenda que la CIJ debiera tener las competencias necesarias, ${ }^{44}$ la realidad es que, primero, resulta improbable que la CIJ obtenga estas facultades y, segundo, que tampoco parece claro que de tal manera pueda cambiar la mentalidad de la CIJ hacia una coordinación entre regímenes.

Incluso si consideramos al artículo 103 de la Carta de las Naciones Unidas, como un tipo de disposición constitucional que establece una primacía de las Naciones Unidas sobre otras obligaciones de derecho internacional público, parece impensable que de esta manera se establezca una supremacía coordinativa sobre otros regímenes del derecho internacional. ${ }^{45}$ Como

44 Scheinin, Martin, “The ICJ and the Individual”, International Community Law Review, vol. 9, núm. 2, 2007, pp. 123-137, en especial p. 123.

45 Koskenniemi, Martti y Leino, Päivi, "Fragmentation of International Law? Postmodern Anxieties”, Leiden Journal of International Law, vol. 15, núm. 3, 2002, pp. 553-579, en especial 
hemos señalado con anterioridad, los casos Kadi del Tribunal de Justicia de la Unión Europea, en las que rechazó de facto la primacía de resoluciones del Consejo de Seguridad sobre las disposiciones en materia de derechos humanos, son una buena muestra de ello. ${ }^{46}$

Conviene afirmar que un rol de coordinación entre regímenes de diferente temática también podrán desempeñarlo la CVDT y el régimen de tratados internacionales como elemento generalmente aceptado dentro del derecho internacional formal-procesal. Si bien el preámbulo y los artículos 3o. y 4o. de la CVDT sugieren lo contrario — y a pesar de que países importantes como Estados Unidos y Francia todavía no han ratificado la Convención de Viena - está de cualquier modo aceptado que la CVDT, a grandes rasgos, refleja y codifica el derecho consuetudinario en la materia. ¿Hasta qué medida una norma de la CVDT refleja costumbre? Ello debe determinarse caso por caso. ${ }^{47}$ Sin embargo, la CVDT refleja el derecho consuetudinario de la década de 1960, y teniendo en cuenta que los nuevos elementos de especialización y fragmentación del derecho internacional público, que también se empezaron a desarrollar desde los cincuenta y sesenta, no se reflejan en la Convención de Viena.

En este mismo sentido, el artículo 30 de la CVDT se considera como la codificación del principio general de lex posterior, lex superior y pacta tertii. En su parágrafo 3 se enfoca a aquellos casos en los cuales las partes de un primer tratado también son partes de un tratado subsecuente, el cual contradice el primero. En este caso, prevalece el tratado posterior. Sin embargo, esta constelación no es aplicable a la relación entre la OMC y otros regímenes especializados, dado que es prácticamente imposible que todos los miembros de la OMC sean partes de un tratado, posterior a su fundación en 1995.

Consecuentemente, el artículo 30, parágrafo 4, de la CVDT dispone el caso mucho más probable: que no todas las partes de un tratado anterior forman parte de un tratado posterior. En este supuesto, se debe aplicar el

p. 559; Zemanek, Karl, The Legal Foundations of the International System: General Course on Public International Law, Leiden, Martinus Nijhoff, 1997, pp. 231 y 232.

46 Tribunal de Justicia de la Unión Europea, Yassin Abdullah Kadi y Al Barakaat International Foundation vs. Council and Commission, (Kadi I), cit., parás. 314 y 326; Tribunal de Justicia de la Unión Europea, Yassin Abdullah Kadi y Al Barakaat International Foundation vs. Council and Commission, (Kadi II), cit., pará. 133.

47 Aust, Anthony, "Vienna Convention on the Law of Treaties (1969)", en Wolfrum, Rüdiger (comp.), The Max Planck Encyclopedia of Public International Law, Oxford, Oxford University Press, 2012, vol. 10, pp. 709-714, pará. 14. 
nuevo tratado entre sus partes, mientras que el tratado viejo sigue vigente entre las demás partes. En resumen, se trata de la codificación del principio lex posterior derogat legi priori.

Con base en el artículo 31 de la CVDT, un tratado debe interpretarse de buena fe de acuerdo con su significado habitual y de conformidad con sus disposiciones, así como a su contexto. Es este principio el que explica por qué diversos mecanismos de solución de controversias en regímenes diferentes, ante hechos similares, concluyen en razonamientos jurídicos distintos. ${ }^{48}$ En este sentido, la $\mathrm{OMC}$ ha desarrollado su propia práctica específica relacionada al artículo 31 de la CVDT y, dentro del propio régimen de la OMC, el Órgano de Apelaciones se caracteriza por una interpretación flexible. ${ }^{49}$ Todas las excepciones al derecho de la OMC, como la protección del medio ambiente con base en el artículo XX del GATT, requieren, de acuerdo con los criterios del Órgano de Apelaciones, un análisis específico para cada caso. ${ }^{50}$

En este contexto, hasta ahora la OMC ha sido renuente en la aplicación del derecho internacional externo en su propio sistema legal con base en el artículo 31 de la CVDT. Tanto en los casos de Biotecnología ${ }^{51}$ como en los casos del atún, ${ }^{52}$ los paneles y el Órgano de Solución de Controversias exigieron que los tratados externos deberían estar abiertos a todos los miembros de la OMC. Las interpretaciones de la OMC se fundamentan en el artículo 30 de la Convención de Viena, las cuales — en la práctica — son imposibles de cumplir. En consecuencia, la CVDT y sus reglas de interpretación de los tratados no pueden desempeñar una función de coordinación efectiva entre el derecho de la OMC y los regímenes externos.

Finalmente, el artículo 41 de la CVDT ofrece la posibilidad de que dos partes puedan enmendar las disposiciones relevantes de un tratado multilateral, siempre y cuando ello no resulte incompatible con el objetivo y el tratado multilateral en su conjunto. Sin embargo, el objetivo principal de la

48 Van Damme, Isabelle, op. cit., p. 213.

49 Ibidem, pp. 216 y 221.

50 World Trade Organization, United States - Standards for Reformulated and Conventional Gasoline, Report of the Appellate Body, adopted 20.11.1996, WT/DS2/AB/R, pará. 18.

51 World Trade Organization, European Communities - Measures Affecting the Approval and Marketing of Biotech Products, Panel Report, adopted 21.11.2006, WT/DS291/R, Add. 1 to Add. 9 and Corr. 1 / WT/DS292/R, Add. 1 to Add. 9 and Corr. 1 / WT/DS293/R, Add. 1 to Add. 9 and Corr. 1, 847.

52 World Trade Organization, United States - Measures Concerning the Importation, Marketing and Sale of Tuna and Tuna Products, cit. 
OMC es la liberalización amplia, integral y universal, y por lo tanto igualitaria, del comercio mundial. ${ }^{53}$ Además, la propia OMC estipula reglas que permiten a sus miembros la profundización de su integración económica mediante acuerdos plurilaterales o, en su caso, comerciales regionales. También, desde este punto de vista, la aplicación del artículo 41 de la CVDT es impensable.

De todo lo anterior, podemos aseverar que la CVDT no contiene los elementos necesarios para resolver conflictos entre regímenes especializados. En sus reglas de interpretación y resolución de conflictos se limita demasiado a secuencias cronológicas, es decir, al principio de lex posterior. Con una plena aplicación de la CVDT, no sólo se tendría que tomar en cuenta la cronología de diferentes obligaciones multilaterales por parte de los Estados, sino también sus respectivas reservas en el sentido del artículo 18 de la CVDT. El resultado sería un auténtico mosaico heterogéneo, ${ }^{54}$ el cual pondría en peligro el desarrollo universal y homogéneo de la OMC y los demás regímenes especializados, lo cual seguramente no representó la intención de los fundadores de tales regímenes.

De ello podemos deducir que las reglas de interpretación de los artículos 30 y 31 de la CVDT todavía consideran al derecho internacional público como un sistema homogéneo y lineal, cuya función se limita a la coordinación entre Estados, sin tener en cuenta la especialización del derecho internacional mediante regímenes especializados.

\section{2. ¿Coordinación a base de elementos procesales nacionales?}

Resulta interesante señalar que la coordinación que no proporciona la CVDT también pudiera tener su fundamento en el derecho internacional no contractual, ya sea a través de la costumbre, o bien mediante los principios generales de derecho. Si bien la CVDT no contempla norma alguna que se refiera de manera específica a la especialización, el principio de lex

53 El acuerdo que establece la OMC define sus objetivos de la siguiente manera: "desarrollar un sistema de comercio multilateral integrado, más viable y duradero", así como "preservar los principios básicos y promover los objetivos subyacentes a este sistema comercial multilateral”. Acuerdo de Marrakech por el que se establece la Organización Mundial del Comercio, 15 de abril de 1994, 1867 UNTS 154.

54 Böckenförde, Markus, op. cit., p. 985. 
specialis goza de una amplia aceptación, tanto en la práctica como en la jurisprudencia del derecho internacional. Ya antes de la entrada en vigor de la Convención de Viena, el juez Hsu sustentó en su opinión disidente en el caso Ambatielos de la CIJ que una norma específica debe prevalecer sobre una norma general..$^{55}$ En el caso Gabčíkovo-Nagymaros, la misma CIJ determinó que un tratado celebrado entre Hungría y Checoslovaquia en 1977 constituía lex specialis frente a las normas generales de responsabilidad del Estado ${ }^{56}$ En términos similares, la CIJ se expresó en el caso Nicaragua vs. Estados Unidos. ${ }^{57}$ En su decisión de INA Corporation vs. Iran, el tribunal de reclamos entre Estados Unidos calificó un tratado de amistad lex specialis sobre las normas generales de compensaciones entre Estados. ${ }^{58}$

Sin embargo, la relación específica entre las clásicas reglas de lex posterior - tal como la contempla el artículo 30 de la CVDT - y lex specialis, hasta la fecha, no ha tenido un papel trascendental en decisiones de derecho internacional y, consecuentemente, no existe jurisprudencia de los diferentes organismos de solución de controversias entre el artículo 30 de la CVDT y el principio de lex specialis. Al final, los límites del artículo 30 de la CVDT dependen del alcance de estos principios dentro del derecho internacional público general. ${ }^{59} \mathrm{Si}$ bien la CVDT por sí sola no puede ofrecer una solución satisfactoria para la relación de conflictos entre regímenes, la gran mayoría de los internacionalistas está de acuerdo en que tal solución debe basarse en el derecho internacional general. ${ }^{60}$ Ello resulta por demás lógico, en el sentido de considerar que una separación estricta entre regímenes sería artificial y, por tanto, no reflejaría la realidad de cooperación entre Estados. Más bien,

55 Corte Internacional de Justicia, Ambatielos Case Jurisdiction (Greece vs. United Kingdom), ICJ Reports, 1952, 28 (44).

56 Corte Internacional de Justicia, Case concerning the Gabčíkovo-Nagymaros Project (Hungary vs. Slovakia), Judgment of 25.9.1997, ICJ Reports, 1997, pp. 7 y 76, pará. 132.

57 Corte Internacional de Justicia, Case concerning Military and Paramilitary Activities in and against Nicaragua (Nicaragua vs. United States of America), Judgement of 27.6.1986, ICJ Reports, 1986, p. 14, pará. 274 .

58 Iran-US Claims Tribunal, INA Corporation vs. Iran, 12 de agosto de 1985, parás. 23 y 24, disponible en: https: / /jusmundi.com/en/document/decision/en-ina-corporation-v-the-governmentof-the-islamic-republic-of-iran-award-award-no-184-161-1-tuesday-13th-august-1985\#decision_ 4872 (fecha de consulta: 14 de abril de 2020).

59 Orakhelashivili, Alexander, “Article 30 Convention of 1969”, en Corten, Olivier y Klein, Pierre (comps.), The Vienna Conventions on the Law of Treaties: A Commentary, Oxford, Oxford University Press, 2011, pp. 764-803, pará. 22.

60 Simma, Bruno y Pulkowski, Dirk, op. cit., p. 502. 
hemos de asumir que los Estados quieren desarrollar varios temas de manera paralela, por lo que se requiere un marco común para su coordinación. ${ }^{61}$

Así pues, los regímenes modernos de derecho internacional generalmente están diseñados como acuerdos marco, donde su contenido sustantivo puede ser cambiado o reformado sin procedimientos formales, o, en el mejor de los casos, con procedimientos simplificados. ${ }^{62}$ Ejemplos de ellos son el Acuerdo de la $\mathrm{OMC},{ }^{63}$ en cuyo marco se desarrollan las diferentes áreas de la liberalización del comercio; el Acuerdo de las Naciones Unidas sobre el cambio climático, ${ }^{64}$ así como los instrumentos de protección regional y universal de los derechos humanos, que evolucionan a través de varios protocolos. En tal contexto, las reglas temporales del artículo 30 (3) y (4) de la CVDT no resultan adecuadas para regular efectivamente la coexistencia pacífica entre regímenes especializados que se desarrollan en paralelo.

Es bien sabido que las normas de conflicto son una parte integral de los ordenamientos legales nacionales, las cuales en países que se basan en derecho civil se rigen a través de los principios res iudicata y lis pendens. Básicamente, la idea es que una controversia ya decidida (res iudicata), o pendiente para una decisión (lis pendens), no puede ser presentada de nueva cuenta en otro foro adicional. Éstos constituyen principios de derecho civil, cuyo objetivo es garantizar la previsibilidad y la seguridad jurídica, buscando evitar litigios paralelos.

Se ha aceptado generalmente que el principio de res iudicata forma parte del derecho internacional público como principios generales de derecho nacional, y al respecto también existen numerosos ejemplos en la práctica judicial internacional. Podemos citar algunos de ellos respecto a la CIJ: el caso del laudo arbitral entre Honduras y Nicaragua, ${ }^{65} \mathrm{o}$ el caso de Límite Territorial y Marítimo entre Camerún y Nigeria. ${ }^{66}$ También existen otros ejemplos en

61 Pauwelyn, Joost, "Bridging Fragmentation...”, op. cit., pp. 903 y 904.

62 Pauwelyn, Joost, “The Role of Public...”, op. cit., p. 545.

63 Acuerdo de Marrakech por el que se establece la Organización Mundial del Comercio, 15 de abril de 1994, 1867 UNTS 154.

64 Convención Marco de las Naciones Unidas sobre el Cambio Climático, 9 de mayo de 1992, 1771 UNTS 107.

65 Corte Internacional de Justicia, Case concerning the Arbitral Award made by the King of Spain on 23 December 1906 (Honduras vs. Nicaragua), ICJ Reports, 1960, p. 192.

66 Corte Internacional de Justicia, Case concerning Land and Maritime Boundary between Cameroon and Nigeria (Cameroon vs. Nigeria), Preliminary Objections, Judgment, ICJ Reports, 2002, p. 303. 
foros de resolución de disputas judiciales ${ }^{67}$ Sin embargo, y de acuerdo con Pauwelyn y Salles, la OMC es muy renuente para aplicar el principio de res iudicata. ${ }^{68}$ Esto resulta un tanto incomprensible, ya que su uso está sujeto a los criterios estrictos de coincidencia de partes, objeto y causa de acción. ${ }^{69}$

Conviene subrayar que ambos principios de res iudicata y lis pendens se encuentran reflejados en los tratados del régimen de protección de derechos humanos. Casi todos los órganos de derechos humanos niegan su jurisdicción si una queja de un individuo ya fue resuelta (res iudicata) o está pendiente (lis pendens) en otro organismo internacional de derechos humanos. ${ }^{70}$

En claro contraste al principio de res iudicata, no existe jurisprudencia en derecho internacional sobre el principio de lis pendens. Consecuentemente, en la correspondiente doctrina existen voces de diverso tipo (de apoyo, ${ }^{71}$ indecisas $^{72}$ y negativas $)^{73}$ con respecto a la idea de considerar a lis pendens como parte del derecho internacional público. En la opinión de Cuniberti — en la que niega tal postura - se basa, particularmente, en el argumento de que el principio de lis pendens constituye un principio propio del derecho civil, el cual no resulta aplicable en partes importantes del mundo y, por lo tanto,

67 Reinisch, August, "The Use and Limits of Res Judicata and Lis Pendens as Procedural Tools to Avoid Conflicting Dispute Settlement Outcomes", The Law and Practice of International Courts and Tribunals, vol. 3, núm. 1, 2004, pp. 37-77, en especial pp. 44-48.

68 Pauwelyn, Joost y Salles, Luiz Eduardo, "Forum Shopping before International Tribunals: (Real) Concerns, (Im)Possible Solutions”, Cornell International Law Journal, vol. 42, 2009, pp. 77-118, en especial pp. 102 y 103.

${ }^{69}$ Corte Permanente de Justicia Internacional, Interpretation of Judgments Nos. 7 and 8 (Germany vs. Poland), 1927 P.C.I.J. (ser. A) No. 13 (Dec. 16), Dissenting opinion of judge Anzilotti; Shany, Yuval, The Competing Jurisdictions of International Courts and Tribunals, Oxford, Oxford University Press, 2003, pp. 22 y 23; World Trade Organization, India - Measures Affecting the Automotive Sector, Report of the Panel, adopted 21.12.2001, WT/DS146/R, WT/ DS175/R, pará. 7.65 .

70 Véase el artículo 35, parágrafo 2, letra $b$, del Convenio Europeo para la Protección de los Derechos Humanos y de las Libertades Fundamentales, 3 de septiembre de 1953, 213 UNTS 222, o el artículo 44 de la Convención Americana sobre Derechos Humanos, 22 de noviembre de 1969, 1144 UNTS 123.

71 Reinisch, August, op. cit., p. 48.

72 Shany, Yuval, "The First MOX Plant Award: The Need to Harmonize Competing Environmental Regimes and Dispute Settlement Procedures", Leiden Journal of International Law, vol. 17, núm. 4, 2004, pp. 815-827.

73 Cuniberti, Gilles, "Parallel Litigation and Foreign Investment Dispute Settlement”, ICSID Review Foreign Investment Law Journal, vol. 21, 2006, pp. 381-429, en especial pp. 419 y 420. 
no puede ser un principio general de derecho en el ámbito internacional público. Sin embargo, su argumento más relevante es que ambos principios - res iudicata y lis pendens - pueden aplicarse para tribunales igualmente competentes desde el punto de vista sustantivo, aun cuando se encuentren en lugares separados. ${ }^{74}$ No obstante, ello no es el caso entre regímenes especializados, los cuales precisamente se distinguen por diferencias y distintos puntos de vista en el derecho material.

Por su parte, otros sistemas jurídicos se enfrentan a dicha problemática con instrumentos jurídicos muy diferentes y, por ende, no pueden constituir un principio general de derecho. ${ }^{75}$ Pero si su fundamento se encuentra en los principios generales de derecho, o bien en una práctica reiterada de los sujetos de derecho internacional — probablemente emergente como derecho consuetudinario_- tiene más importancia teórica que relevancia práctica. Al final, se tratará de principios legales de derechos nacionales procesales que podrían establecerse como costumbre internacional. Es inherente a los sistemas de derecho civil que, con base en los principios de res iudicata y lis pendens, el primer tribunal materialmente competente debe tener jurisdicción. De esta manera se evitan litigios dobles. Finalmente, se trata de una especie de batalla en dónde se constituye primeramente jurisdicción. ${ }^{76}$

\section{Common law}

En contraste, el common law parece ser más adecuado a las características de derecho internacional público. El derecho internacional moderno y especializado permite —al igual que el common law_, en principio, litigios paralelos y, de la misma manera, se basa en partes muy importantes de su desarrollo e interpretación en su jurisprudencia.

Es cierto que el common law, como principio general de derecho, tiene menos aceptación que el derecho civil. No obstante, aquél es el sistema legal único en un total de 23 países, incluyendo a Estados Unidos, Gran Bretaña, Canadá, Australia y Nueva Zelanda. Además, también existen sistemas mix-

74 Ibidem, p. 383.

75 Idem.

76 Brand, Ronald A., "Forum Non Conveniens", en Wolfrum, Rüdiger (comp.), The Max Planck Encyclopedia of Public International Law, Oxford, Oxford University Press, 2012, vol. 4, pp. 198-203, en especial p. 202. 
tos, por lo que el common law se aplica, al menos parcialmente, en un tercio de los Estados del ordenamiento jurídico nacional. ${ }^{77}$

A pesar de ello, es cuestionable si el common law pueda tener su fundamento exclusivamente a base de principios generales de derecho. Sin embargo, las figuras de equity ${ }^{78}$ y estoppel ${ }^{79}$ son ejemplos de figuras generalmente aceptadas en derecho internacional público, que tienen sus orígenes en el common law. En última instancia, la validez del common law para la coordinación en derecho internacional debe buscarse en el emergente derecho consuetudinario hacia una interpretación armoniosa entre regímenes especializados, en conjunto con los principios nacionales de derecho.

Sistemas de derecho civil, mediante los principios de res iudicata y lis pendens, intentan establecer jurisdicción en el primer tribunal y evitar una litigación paralela. Se trata de una carrera hacia dónde se puede establecer primero jurisdicción. ${ }^{80}$ En contraste, en el common law, el factor determinante no es el primer establecimiento de jurisdicción, sino más bien en qué foro se emite primero una decisión. ${ }^{81}$ Se trata de una "carrera" hacia dónde se obtiene una sentencia. Un elemento procesal de carácter esencial del common law es el forum non conveniens, ${ }^{82}$ el cual permite a jueces rechazar un caso y referirlo a otro foro. Ello otorga a los tribunales - como es inminente en el common law - un mayor margen de discrecionalidad, en caso de que existiera otro para la resolución del caso.

El forum non conveniens es parte de todos los sistemas del common law, pero su aplicación e interpretación muestran diferencias en las distintas jurisdic-

7763 de 193 miembros de las Naciones Unidas contemplan, al menos parcialmente, elementos del common law. Disponible en: http://www.juriglobe.ca/eng/syst-onu/rep-sys-juridique. php\#syst2 (fecha de consulta: 29 de agosto de 2020). También es importante mencionar que tanto en Estados Unidos como en Canadá existen excepciones locales (Luisiana y Quebec, respectivamente) que contienen sistema de derecho civil.

78 Francioni, Francesco, “Equity in International Law”, en Wolfrum, Rüdiger (comp.), The Max Planck Encyclopedia of Public International Law, Oxford, Oxford University Press, 2012, vol. 3, pp. 632-642.

79 Cottier, Thomas y Müller, Jörg Paul, “Estoppel”, en Wolfrum, Rüdiger (comp.), The Max Planck Encyclopedia of Public International Law, Oxford, Oxford University Press, 2012, vol. 3, pp. 671-677.

80 Brand, Ronald A., op. cit., p. 202.

81 Idem.

82 Ibidem, p. 198. Una formulación similar se encontraba hasta su reforma en el artículo 9o., parágrafo 3, de la Ley de Jurisdicción Suiza (Schweizer Gerichtsstandgesetz). 
ciones. Las interpretaciones estadounidenses e inglesas más extensas del forum non conveniens tienen por objeto remitir el proceso al foro que parece ser el más adecuado para la resolución del caso. Se utiliza una ponderación de criterios públicos y privados, lo que significa que el caso puede tratarse mejor en interés de todas las partes y en interés de la justicia. ${ }^{83}$ En el caso Spiliada, un tribunal inglés definió los elementos más importantes para poder utilizar el forum non conveniens. Estos criterios fueron acatados por la mayoría de los países del common law. De acuerdo con ello, no sólo se debe demostrar que Inglaterra es un foro inadecuado para decidir el caso, sino que claramente existe un foro más apropiado. La carga de la prueba recae en el demandante; pero si el tribunal estima que prima facie existe tal foro, tal carga probatoria se traslada hacia el oponente. ${ }^{84}$

Por consiguiente, la idea del common law es que los casos sean decididos por el tribunal materialmente más cercano, mientras que los sistemas de derecho civil ponen más relieve en la previsibilidad y efectividad del proceso. Mientras que los principios de res iudicata y lis pendens se enfocan principalmente a criterios temporales, el forum non conveniens se concentra en el foro más especializado. Es esta discrecionalidad de jueces ante la especialidad de posibles foros, la cual parece ser más apropiada para la coordinación de un derecho internacional público moderno. El juez Antonin Scalia, en su opinión mayoritaria en el caso American Dredging Co. vs. Miller de la Suprema Corte de Estados Unidos, señaló claramente que la doctrina de forum non conveniens busca evitar la posibilidad de forum shopping. ${ }^{85}$

Aún no queda del todo claro cómo la coordinación entre regímenes hegemónicos y jerárquicamente iguales se debe definir en la práctica cotidiana. Los principios nacionales, cuya tarea es evitar conflictos entre diferentes sistemas jurídicos a nivel doméstico y transnacional, pudieran constituirse como base. Dadas las características específicas del derecho internacional público, la figura de forum non conveniens del common law pareciera la más apropiada, y no sus correspondientes figuras de sistemas civiles.

Hasta la fecha, en el derecho internacional no existe jurisprudencia específica sobre el forum non conveniens. El ejemplo más concreto en el derecho

83 Ibidem, p. 200.

84 House of Lords (Reino Unido), Spiliada Maritime Corp vs. Cansulex Ltd. [1986], UKHL 10, 19 de noviembre de 1986.

85 Suprema Corte de Estados Unidos, American Dredging Co. vs. Miller, 510 U.S. 443 (1994), 23 de febrero de 1994. 
internacional público corresponde al caso MOX-Plant, que implicó una serie de quejas y demandas por parte de Irlanda en contra de una planta nuclear británica en el mar irlandés, cerca de Sellafield. El gobierno irlandés adjudicó el caso a tres órganos de solución de controversias diferentes: primero, con base en el Convenio para la Protección del Medio Marino del Atlántico Nororiental (OSPAR); segundo, con fundamento en la Convención de Naciones Unidas sobre el Derecho del Mar (Convemar), y tercero, en el Tribunal de Justicia por violación del derecho de la Unión Europea. El caso es notablemente llamativo por la decisión procesal del Tribunal establecido con fundamento en el anexo VII de la Convemar. Si bien este Tribunal ha permitido medidas preliminares, también ha suspendido sus propios procedimientos en espera de tener más clara la situación respecto al derecho de la Unión Europea. ${ }^{86}$ Esto es, se retiró voluntariamente porque, en su opinión, elTribunal de Justicia de la Unión Europea se encontraba más cercano al derecho sustantivo y material. Finalmente, el caso fue resuelto por éste.

A todo ello, resulta importante destacar que el procedimiento del Tribunal del anexo VII es similar, por lo tanto, a la figura nacional del forum non conveniens; incluso, sus criterios nacionales del common law no resultan directamente aplicables a la lógica y las realidades del derecho internacional. Esta interpretación es, de igual modo, consistente con la figura de derecho material de lex specialis, y parece ser adecuada para regímenes especializados. En ambos casos prevalecen reglas especializadas sobre la aplicación de criterios con una estricta secuencia temporal. Sin embargo, el diseño exacto de estas reglas — requiere, en principio, la aceptación de todos los regímenes especializados — debe definirse por la práctica emergente en el derecho internacional y sus correspondientes contribuciones científicas.

Sin embargo, y dado que el derecho internacional público reconoce los principios básicos del derecho civil, esto también puede aplicarse a sus correspondientes principios del common law.

Todos estos principios de derecho civil tienen como objetivo realizar una función de coordinación y equilibrio entre diferentes foros y sistemas legales. En este sentido, las preguntas que corresponden realizarse serían las siguientes: en qué medida estos principios pueden integrarse en las realidades del derecho internacional, y en qué medida los Estados y órganos de reso-

86 Churchill, Robin R., "MOX Plant Arbitration and Cases”, en Wolfrum, Rüdiger (comp.), The Max Planck Encyclopedia of Public International Law, Oxford, Oxford University Press, 2012, vol. 7, pp. 403-410. 
lución internacional de controversias internacionales especializados aplican, adaptan y desarrollan dichos principios en la práctica.

\section{A MANERA DE CONCLUSIÓN}

Como sabemos, el derecho internacional contemporáneo se caracteriza por su especialización. Sus regímenes, como el derecho comercial internacional, la protección internacional de derechos humanos o el derecho ambiental internacional, son sólo los ejemplos más conocidos de su fragmentación. Muchos de estos regímenes establecen sus propios órganos de solución de controversias, los cuales se caracterizan por su reclamo de hegemonía.

Cabe mencionar que, hasta la fecha, en materia de derechos humanos no ha existido mayor acercamiento hacia el derecho de la OMC u otros regímenes; pero debido a su característica, resulta indudable que prevalecería su propio sistema. En suma, y hasta ahora, los organismos externos de solución de controversias, sin tener obligación expresa, han hecho referencia a la OMC en aproximadamente 150 ocasiones. La principal razón de ello es la práctica de garantizar una interpretación armoniosa del derecho internacional. El mismo principio se puede observar en casos del derecho ambiental internacional, a pesar de que sus referencias hacia otros regímenes se limitan a opiniones de carácter concurrente o disidente.

Todo lo antes señalado supone una necesaria coordinación entre regímenes, la cual sólo puede proporcionar el derecho internacional público general. No obstante, ni las Naciones Unidas ni la CIJ y tampoco la CVDT cuentan con la calidad y las características para proporcionar dicha coordinación. Es imposible establecer jurisdicción ante la CIJ con la participación de áreas comerciales o incluso individuos, tal y como lo hacen los regímenes del derecho internacional moderno. Adicionalmente, ésta carece de una aceptación universal, ya que sólo una tercera parte de los Estados reconoce plenamente su jurisdicción. Aunque la CVDT es considerada - por lo menos parcialmente - una constitución de materia procesal del derecho internacional, sus disposiciones carecen de elementos suficientes que pudieran resolver conflictos entre regímenes especializados. Hemos de recordar que la CVDT fue negociada antes del advenimiento de regímenes especializados en el derecho internacional y, en consecuencia, se enfoca a criterios temporales, y no ya a resolver conflictos de especialidad. 
Hay que hacer notar que una posible coordinación entre regímenes especializados se pudiera lograr a través del principio de una interpretación armoniosa del derecho internacional. Este principio parece ser reconocido en todos los regímenes especializados y podría consolidarse como derecho consuetudinario. De la misma manera, se pudiera fundamentar una base legal en los derechos procesales nacionales que, como principios generales de derecho, resultarían igualmente aplicables en derecho internacional público. Sin embargo, el common law pareciera más adecuado que los correspondientes principios del derecho civil, los cuales, al igual que en la CVDT, se enfocan a criterios temporales. El forum conveniens - figura clave en el common law - parece ser un elemento adecuado para brindar coordinación en regímenes especializados.

El caso MOX-Plant entre Irlanda y Gran Bretaña muestra, en la práctica, cómo podría desarrollarse una coordinación entre diferentes regímenes. En ello, el Tribunal Internacional de Derecho del Mar renunció a la aplicación de su jurisdicción, dado que consideró igualmente competente al Tribunal de Justicia de la Unión Europea, estimándolo más cercano al derecho sustantivo. Por último, podemos concluir que se trata de la aplicación de un principio semejante al forum non conveniens. Sin embargo, en la medida en que este principio sea aplicado en la solución de controversias de regímenes especializados y, por tanto, pueda brindar un papel de coordinación, ha de mostrar su futura práctica en el derecho internacional, así como su correspondiente discusión científica doctrinal.

\section{BIBLIOGRAFÍA}

Aust, Anthony, "Vienna Convention on the Law of Treaties (1969)", en Wolfrum, Rüdiger (comp.), The Max Planck Encyclopedia of Public International Law, Oxford, Oxford University Press, 2012, vol. 10.

BÖCKENFÖRDE, Markus, “Zwischen Sein und Wollen - Über den Einfluss umweltvölkerrechtlicher Verträge im Rahmen eines WTO-Streitbeilegungsverfahrens", Zeitschrift für allgemeines öffentliches Recht und Völkerrecht, vol. 63, 2003.

Bradford, Anu, "Regime Theory", en Wolfrum, Rüdiger (comp.), The Max Planck Encyclopedia of Public International Law, Oxford, Oxford University Press, 2012, vol. 8. 
Brand, Ronald A., "Forum Non Conveniens", en Wolfrum, Rüdiger (comp.), The Max Planck Encyclopedia of Public International Law, Oxford, Oxford University Press, 2012, vol. 4.

Churchill, Robin R., "MOX Plant Arbitration and Cases", en Wolfrum, Rüdiger (comp.), The Max Planck Encyclopedia of Public International Law, Oxford, Oxford University Press, 2012, vol. 7.

COMISIÓn DE DERECHO INTERNACIONAL, "Fragmentation of International Law: Difficulties Arising from the Diversification and Expansion of International Law", Report of the Study Group of the International Law Commission, A/CN.4/L.682, 13 de abril de 2006.

COTTIER, Thomas y MüLler, Jörg Paul, “Estoppel”, en Wolfrum, Rüdiger (comp.), The Max Planck Encyclopedia of Public International Law, Oxford, Oxford University Press, 2012, vol. 3.

CuniberTi, Gilles, "Parallel Litigation and Foreign Investment Dispute Settlement”, ICSID Review Foreign Investment Law Journal, vol. 21, 2006.

FASSBENDER, Bardo, "The United Nations Charter as Constitution of the International Community", Columbia Journal of Transnational Law, vol. 36, 1998.

FisCHER-LesCAnO, Andreas y TeUBner, Gunther, "Regime-Collisions: The Vain Search for Legal Unity in the Fragmentation of Global Law”, Michigan Journal of International Law, vol. 25, 2004.

FRANCIONI, Francesco, "Equity in International Law”, en Wolfrum, Rüdiger (comp.), The Max Planck Encyclopedia of Public International Law, Oxford, Oxford University Press, 2012, vol. 3.

GanTZ, David A., "Dispute Settlement under the NAFTA and the WTO: Choice of Forum Opportunities and Risks for the NAFTA Parties", American University International Law Review, vol. 14, 1998.

HAFNER, Gerhard, "Pros and Cons Ensuing from Fragmentation of International Law”, Michigan Journal of International Law, vol. 25, 2004.

HARRISON, James, The Human Rights Impact of the World Trade Organisation, Portland, 2007.

JOSEPH, Sarah, Blame it on the WTO?:A Human Rights Critique, Oxford, Oxford University Press, 2011.

Koskenniemi, Martti y LeINO, Päivi, "Fragmentation of International Law? Postmodern Anxieties", Leiden Journal of International Law, vol. 15, núm. 3, 2002. 
KuO, Ming-Sung, "Between Fragmentation and Unity: The Uneasy Relationship between Global Administrative Law and Global Constitutionalism”, San Diego International Law Journal, vol. 10, 2008.

LIXINSKI, Lucas, "Treaty Interpretation by the Inter-American Court of Human Rights: Expansionism at the Service of the Unity of International Law”, European Journal of International Law, vol. 21, núm. 3, 2010.

MARCEAU, Gabrielle et al., "The WTO's Influence on Other Dispute Settlement Mechanisms: A Lighthouse in the Storm of Fragmentation", Journal of World Trade, vol. 47, núm. 3, 2013.

OraKHElaShivili, Alexander, “Article 30 Convention of 1969”, en CORTEN, Olivier y KLEIN, Pierre (comps.), TheVienna Conventions on the Law of Treaties: A Commentary, Oxford, Oxford University Press, 2011.

PauWELYN, Joost, "Bridging Fragmentation and Unity: International Law as a Universe of Inter-Connected Islands", Michigan Journal of International Law, vol. 25, 2003.

PauWelyn, Joost, "The Role of Public International Law in the WTO: How Far Can We Go?", The American Journal of International Law, vol. 95, núm. 3, 2001.

PAUWELYN, Joost y SALLES, Luiz Eduardo, "Forum Shopping before International Tribunals: (Real) Concerns, (Im)Possible Solutions", Cornell International Law Journal, vol. 42, 2009.

Petersmann, Ernst-Ulrich, The GATT/WTO Dispute Settlement System: International Law, International Organizations and Dispute Settlement, Leiden, Martinus Nijhoff, 1997.

POSNER, Eric A., "The Decline of the International Court of Justice”, Olin Program in Law and EconomicsWorking Paper No. 233, 2004.

ReInISCH, August, "The Use and Limits of Res Judicata and Lis Pendens as Procedural Tools to Avoid Conflicting Dispute Settlement Outcomes", The Law and Practice of International Courts and Tribunals, vol. 3, núm. 1, 2004. SCHEININ, Martin, "The ICJ and the Individual”, International Community Law Review, vol. 9, núm. 2, 2007.

SHany, Yuval, The Competing Jurisdictions of International Courts and Tribunals, Oxford, Oxford University Press, 2003.

SHANY, Yuval, "The First MOX Plant Award: The Need to Harmonize Competing Environmental Regimes and Dispute Settlement Procedures", Leiden Journal of International Law, vol. 17, núm. 4, 2004. 
Esta revista forma parte del acervo de la Biblioteca Jurídica Virtual del Instituto de Investigaciones Jurídicas de la UNAM

Simma, Bruno y PULKOwski, Dirk, "Of Planets and the Universe: Self-Contained Regimes in International Law”, European Journal of International Law, vol. 17, núm. 3, 2006.

VAN Damme, Isabelle, Treaty Interpretation by theWTO Appellate Body, OxfordNueva York, Oxford University Press, 2009.

VRANES, Erich, "Lex superior, lex specialis, lex posterior - Zur Rechtsnatur der «Konfliktlösungsregeln»”, Zeitschrift für ausländisches öffentliches Recht und Völkerrecht, vol. 65, 2005.

ZEMANEK, Karl, The Legal Foundations of the International System: General Course on Public International Law, Leiden, Martinus Nijhoff, 1997. 